\title{
Acute chagas` disease and kidney
}

\author{
Gabriel Melo de Oliveira* and Celiton Felizardo Brito \\ Laboratório de Biologia Celular, Instituto Oswaldo Cruz/FIOCRUZ, Brazil
}

\begin{abstract}
Chagas' disease, caused by the protozoan Trypanosoma cruzi, where estimated 8 million people are infected in worldwide, mainly in Latin America causing incapacity in infected individuals and more than 10. 000 deaths per year. The pathophysiological mechanisms of the genesis of cardiac involvement, both acute and chronic, are still not completelly understood. However, in experimental models, a relationship was observed between the levels of parasitemia and the high intensity of acute myocarditis with the severity of cardiac symptoms during the chronic phase. Patients with congestive heart failure may present with renal insufficiency and anemia. This triad is denominated the cardio-anemic-renal syndrome. Our results demonstrate that in the early stages of infection there is a transient but marked renal disfunction in mice infected with T. cruzi. In addition, interference in renal/cardiovascular axis, such as the renin angiotensin-aldosterone system block, decreases the mortality of the animals around 30\%. Kidney damage during the infection with T. cruzi is underestimated. Experimental results showed the pathophysiological interconnections with the evolution of acute heart failure. We hope from the results compilation to suggest new diagnostic and therapeutic protocols that minimize cardiac symptomatology during the acute phase and the onset of dilated cardiomyopathy during the chronic phase.
\end{abstract}

\section{Chagas' Disease}

\section{General aspects}

In 1909, Oswaldo Cruz formally announced at the National Academy of Medicine the discovery of American Trypanosomiasis, later called Chagas' disease. This process began in 1907, by the sanitary doctor Carlos Chagas, who was able to discover the parasite, denominated Trypanosoma cruzi. The same way that describe evolutionary cycle, the vector and biological behavior, vertebrate and invertebrate reservoirs, as well as its own disease [1]. An estimated 8 million people are infected with T. cruzi in worldwide, mainly in Latin America where Chagas 'disease remains one of the serius public health problems, causing incapacity in infected individuals and more than 10 . 000 deaths per year. In the past two decades, Chagas ' disease has spread to more uninfected regions compared with its evolution since over 9 . 000 years ago [2]. It's estimated that the incidence is around 700.000 to 800.000 new cases per year and 45.000 patients die due to cardiac complications in the Americas [3,4]. In Brazil, according to the Ministry of Health database (Data/SUS), poverty-related diseases account for 2.19 mortality per 100.000 habitants and where considered the most serious chronic disease in country [5]. Despite the recent notification of the interruption of transmission by the vector Triatoma infestans (one of the hematophagous insect species) in Brazil, other triatomine species continued to transmit the disease [6]. Several occurrences of Chagas' disease have been registered in the Amazon region in the last two decades through serological surveys, isolated case diagnoses or the so-called microepidemias [6,7]. Another important point is the emergence of outbreaks in other locations, both endemic and nonendemic, through infection by ingestion of in natura foods such as: açaí, bacaba (or bacaba-açu, palm native to the Amazon, with fruits that are ingested in natura or as juice) and cane juice. The confirmed cases of oral infection present a severe acute course and with clinical symptomatology differentiated from the classically described $[8,9]$.

\section{Characteristics of $T$. cruzi infection}

The Chagas' disease has as invertebrate vector the triatomine hematophagous insect (popularly known as barber, chupança or procotó) belonging to the order Hemiptera, presenting as the most important from the point of view of transmission the species Triatoma infestans, Triatoma sordida and Panstrongylus megistus (Figure 1). The replicative form of the parasite in the invertebrate host is called the epimastigote and in the vertebrate host it is called the amastigote. The infective form of this protozoan denominated a metacyclic trypomastigote and is found in the final portion of the gut of the insect vector and is released upon defecation that occurs after feeding the insect. The vector transmission of T. cruzi occurs during feeding of the hematophagous insect, which deposits the feces containing the infecting form of the parasite on the skin. Through the lesion caused by the insect bite or by scattering to injured areas or mucous membranes, the parasite reaches the underlying tissue and bloodstream of the host [10].

The main transmission routes of Chagas' disease are:

1) Vetorial: those that are directly linked to the presence of the invertebrate vector, that is, the triatomine;

2) Blood transfusion;

3) Congenital;

4) Oral way through ingestion of contaminated food;

${ }^{*}$ Correspondence to: Gabriel Melo de Oliveira, Lab. Biologia Celular Instituto Oswaldo Cruz, FIOCRUZ, AV. Brasil 4365, Manguinhos, Rio de Janeiro, RJ CEP: 21040-360, Brazil, Tel: +55-21-2562-1366; E-mail: gmoliveira@ioc.fiocruz.br

Key words: trypanosoma cruzi, acute kidney injury, heart failure, I/R AKI

Received: June 29, 2019; Accepted: July 15, 2019; Published: July 18, 2019 

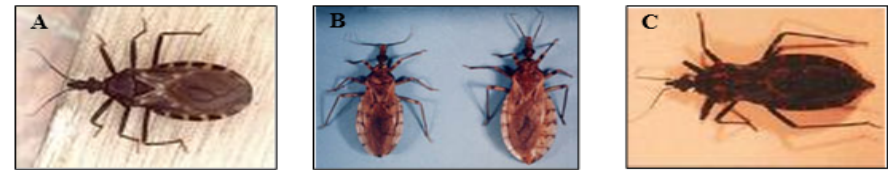

Figure 1. The most important genera of Chagas' disease: (A) Triatoma infestans, characterized by housed with rare wild foci, living in the gaps of the wall, chicken coops and corrals. Triatoma sordida (B) is also a domiciliary species, sometimes located in the peridomicile and in the wild in tree trunks, exposed roots, palms and bromeliads. (C) Panstrongylus megistus is also a domiciliary species found in the peridomicillum in nests of birds and opossums, most common in the northern and northeastern Brazilian states

In according to Jansen 2018, cases/outbreaks occur in distinct regional scenarios, mainly in the Amazon biome and are related to the local interaction mode of humans with their surroundings, as well as with the overall local ecological peculiarities. From 1992 up to 2017, where examined a total of 6587 free-ranging non-volant wild mammal specimens. Results showed that $17 \%$ of mammals were seropositive and $8 \%$ of all animals displayed positive hemocultures indicative of high parasitemia and, consequently, of infectivity potential. We observed that opossums, mainly Philander spp. and Didelphis spp., the coati Nasua nasua, the capuchin monkey Sapajus libidinosus and the golden lion tamarin Leontopithecus rosalia, were mammal taxa that demonstrated higher rates of positive hemocultures. Altogether, suggest that each locality, may display distinct enzootiological and epidemiological scenarios that must be taken into account when it comes to establishing control and/or clarification campaigns of the local population [9].

\section{Clinical Aspects}

\section{Acute Phase}

Chagas' disease presents two clinical forms: acute and chronic. In turn, the chronic phase may be asymptomatic or symptomatic. The acute phase is characterized by being of short duration with clinical symptomatology, usually non-specific and often mild. It may go unnoticed, not reported or incorrectly diagnosed. It promotes 2 to $7 \%$ of mortality, and 8 out of 10 cases present cardiac involvement, acute chagasic myocarditis. At the site of the bite can develop a bulky, erythematous and edematous lesion called "Inoculation Chagoma" and if the infecting material is brought to the eye, there is conjunctivitis with clinical signs, also known as "Romagna's sign", whose duration of more than 30 days allows clear differentiation of viral and bacterial conjunctivitis $[10,11]$.

Main complaints reported by the patient during the acute phase of T. cruzi infection are: prolonged fever, anorexia, adynamia and general malaise. In the physical examination, the increase of lymph node volume and hepatosplenomegaly may be observed. Signs related to heart failure such as fatigue, chest pain and exercise intolerance should be observed. In children, sensory and neurological alterations may occur, indicative of meningoencephalitis and other neurological disorders [10,11].

\section{Diagnosis during the acute phase}

The diagnosis can be made through the set of procedures as:

\section{Anamnesis}

The main question is whether the patient identified the presence of the triatomine (barber) in his home. Correlate social epidemiology as a place of residence in an endemic area, recent trip to the endemic area, relatives or neighbors with similar symptoms, ingestion of fresh food (cane juice, bacaba and especially açaí), contact with vertebrate vectors, mainly opossums and armadillos. Occurrence of blood transfusion or organ transplantation. The incidence of the concomitant occurrence of
Chagas disease and Acquired Immunodeficiency Syndrome (AIDS) has also increased, which has allowed the description of peculiar clinical manifestations [10].

\section{Laboratory}

The hemogram may present a decrease in the hematocrit value, hematimetry and presence or absence of anisocytosis. As for the white series, leukocytosis and increase in the absolute value of lymphocytes can be observed. Contrarily, in some cases we can observe leucopenia and lymphopenia, depending on the stage of infection. There may also be changes in platelet values. In patients with acute myocarditis there is elevation of creatine kinase isotype $\mathrm{MB}$ (CK-MB) levels related to inflammatory myocardial injury $[10,11]$.

\section{Electrocardiography and Echocardiogram}

The ECG presents features compatible with atrioventricular blocks of $1^{\text {st }}, 2^{\text {nd }}$ and $3^{\text {rd }}$ degree, and the second degree is the one with the highest incidence and may be transient. Arritmias, such as bradycardia and sinus tachycardia (depending on the time of infection, since there is oscillation of blood pressure values), ventricular extrasystoles, ventricular tachycardia. We can also observe prolonged and low voltage $\mathrm{T}$ wave, increased $\mathrm{P}$ wave time duration and QT interval. In some cases, diffuse changes in the ST segment and below or supranibly of T. The echocardiography may demonstrate an increase of the chambers (atriums and ventricles), a decrease in the shortening fraction and the ejection fraction $[10,12]$.

\section{Etiologic}

In the acute phase of Chagas' disease, the laboratory diagnosis is based on the observation of the parasite present in the blood of infected individuals. It can be done through direct parasitological tests like fresh blood test, "drop in blade". The direct fresh test is more sensitive than the stained smear and should be the method of choice for the acute phase. If these tests are negative, methods of concentration should be used. Concentration tests (micro-hematocrit or Strout) present 80 to $90 \%$ of positivity and are recommended in the case of a strong suspicion of acute Chagas' disease and negativity of the direct-to-fresh test. The etiological diagnosis can be performed by the presence of antibodies in the serum by means of serological tests, the most used being indirect immunofluorescence (IFI), hemagglutination (HAI) and enzymelinked immunosorbent assay (ELISA) [10,11].

Regarding conventional serological tests, the specificity of the assays has been questioned due to the frequency of infections with other trypanosomatids circulating in the same geographical area as T. cruzi (Leishmania spp and T. rangeli) and are responsible for crossantigenicity and false- positive, and may lead to an incorrect diagnosis and inadequate treatment promoting the worsening of the patient's clinical condition. An accurate diagnosis requires confirmation by parasitological test $[10,13]$.

\section{Chronic Phase}

The chronic phase can be divided into asymptomatic (indeterminate) or symptomatic (cardiac or digestive). In approximately 10 to 20 years, approximately 20 to $30 \%$ of patients surviving the acute phase and asymptomatic carriers of the disease will develop some type of symptomatology. Within this percentage of symptomatic the incidence is $3 \%$ for the neuronal form, $6 \%$ will present the digestive form (megacolon and megaesophagus) and 25 to $30 \%$ will be affected by the cardiac form $[11,14]$. 


\section{Treatment}

The objective of the specific therapy is to act on parasites in the bloodstream or tissues, aiming at the reduction or eradication of parasitemia, thus shortening the symptomatic period of the acute phase of infection and at least theoretically reducing the probability of developing the chronic phase of Chagas' disease. Drugs to date are toxic and require prolonged treatment under careful outpatient medical supervision. They act both in the trypomastigote form, but not in the intracellular amastigotes. However, its use in the chronic phase is still controversial given the uncertainties about its potential to reduce clinical manifestations and the development of dilated chagasic cardiomyopathy $[10,14]$.

The only drug available for treatment is benznidazole (100mg). This drug is a nitroimidazole derivative and is indicated at a dose of 5 to $7 \mathrm{mg} / \mathrm{kg} /$ day for 60 days. Its side effects include rashes after 7 to 10 days of treatment, with increased lymph nodes and fever, indicating discontinuation of treatment. Other effects are peripheral polyneuropathy and granulocytopenia. Therefore, strict clinical followup with weekly blood counts is essential [15].

Monitoring of therapeutic efficacy is based on xenodiagnostic or blood culture results and post-treatment serological reactions. Xenodiagnosis is the most frequently used laboratory resource for the monitoring of parasitological suppression, often only transient, especially when the treatment is done in the indeterminate phase. Treatment in the acute phase or at the beginning of the indeterminate phase generally induces the denial of serodiagnostic tests. However, this does not occur later in the indeterminate phase, or when the manifestations of the chronic phase are already established [10].

\section{Acute renal injury and experimental T. cruzi infection}

Fritz Köberle in 1968 evaluates the pathology of Chagas disease and describes in detail the symptoms of the acute phase, and suggests the relationship between the presence of edema and acute nephritis as described in the respective section: ... an edema of elastic consistency may appear during the second week it may be localized (chiefly in the face) or generalized, sometimes suggesting an acute nephritis, and subsides spontaneously with the other symptoms ... [16]. In addition, some authors describe the disseminated form of experimental infection during the acute phase, but the presence of the parasite in the tissues is predominantly associated with cardiac, skeletal and nervous systems [11].

Kidney involvement due to T. cruzi infection is poorly addressed in the literature, and there is no detailed approach to infection of the parasite in renal tissue. We observed that some studies describe the presence of the parasite in the kidney, even in a small number, but do not address the consequences of this infection in this important vital organ $[17,18]$. Most studies that relate the kidney and Chagas disease point to the issue of organ transplantation $[19,20]$.

In 2004 the emergence of unknown pathogenesis for T. cruzi strains in the Amazon region was described. In a microepidemia, four patients demonstrated severe cardiac involvement and clinical symptomatology that included: sudden fever, myalgia, dyspnea and signs of heart failure. The diagnosis was confirmed by specific tests, especially QBC (Quantitative Buffy Coat) and natural xenodiagnosis. Despite benznidazole treatment, three patients died with severe myocarditis, acute renal failure and cardiac tamponade [21].

Our experimental results clearly demonstrate the marked presence of acute renal injury at the onset of infection and its connection with acute heart failure, forming what we denominated the cardio/renal axis, [22] directly influencing the morbidity/mortality of the disease (Figure. 2).

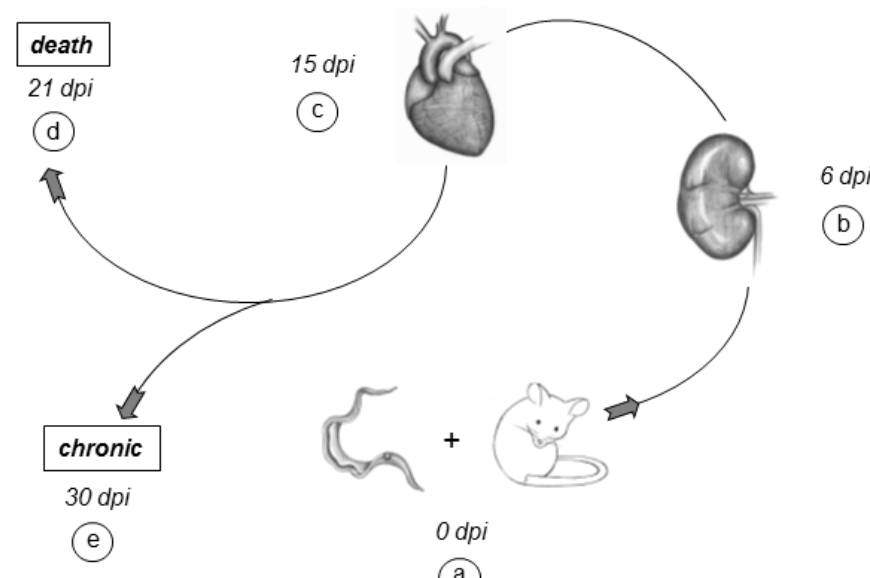

(a)

Figure 2. Progression of experimental infection: Our experimental model, the Balb/c lineage \& Y strain of $T$. cruzi was infected (dpi 0 ) intraperitoneally with high parasite load (a). On the $6^{\text {th }} d p i$, we can see the acute renal injury (b) that articulates the cardio/renal axis and affects the heart, which in turn is at $15^{\text {th }}$ day post infection, relation by the intense acute myocarditis (c). Between 3 and 4 weeks of infection $\left(21^{\text {st }} \mathrm{dpi}\right)$ animals that do not survive will die (d) and survivors, around $20 \%$ progress to the chronic phase $\left(30^{\text {th }} \mathrm{dpi}\right)(\mathrm{e})$

The T. cruzi experimental infection promotes acute renal injury in the early stages of infection, prior to the peak of parasitemia (presence of parasites in the bloodstream) and acute myocarditis. This renal injury is not related to parasite presence or multiplication, but to a decrease in blood flow promoting partial involvement of the proximal tubules, microhemorrhages and capillary congestion [23]. Recently we observed that mesangial cells present high levels of nitric oxide and proinflammatory cytokines in the culture supernatant when infected by T. cruzi. In addition, these cells present less susceptibility to infection, but greater impairment of cell integrity and viability. We believe that nitric oxide (NO), known as a potent microbicide, has an antagonistic role in infection by protecting the cell against parasite invasion and injuring the cell itself [24] (Figure 3). Lemos, et al. in 2013 demonstrated that T. cruzi impairs kidney function, and this impairment is more evident in miceinfected with high parasitic loads. Moreover, these data suggest that, in addition to the extensively studied cardiovascular effects, renal injury should be regarded as an important indicator for better understanding the pan-infectivity of the parasite and consequently for understanding the disease in experimental models [25].

In this way, we suggest in relation to renal injury and T. cruzi infection:

1) Patients in endemic regions or with the possibility of infection by the parasite are evaluated renal parameters such as urea and creatinine in the plasma and measured the glomerular filtration rate (creatinine clearance for 24 hours).

2) A rapid and accurate differential diagnosis (in relation to other anthropozoonoses, such as Leishmania spp) is performed. The closer the infection, the greater the probability of observing the presence of renal impairment.

3) Dosing of electrolytes such as sodium and potassium, assessing the presence of anaemia and the presence of comorbidities, mainly arterial hypertension.

We emphasize that during the hospital routine approximately $67 \%$ of the requests for hospital exams do not present requisition for creatinine and that in $18 \%$ of the cases it is requested only to realize once the dosage of this lesion marker tissue. In the case of Chagas' 

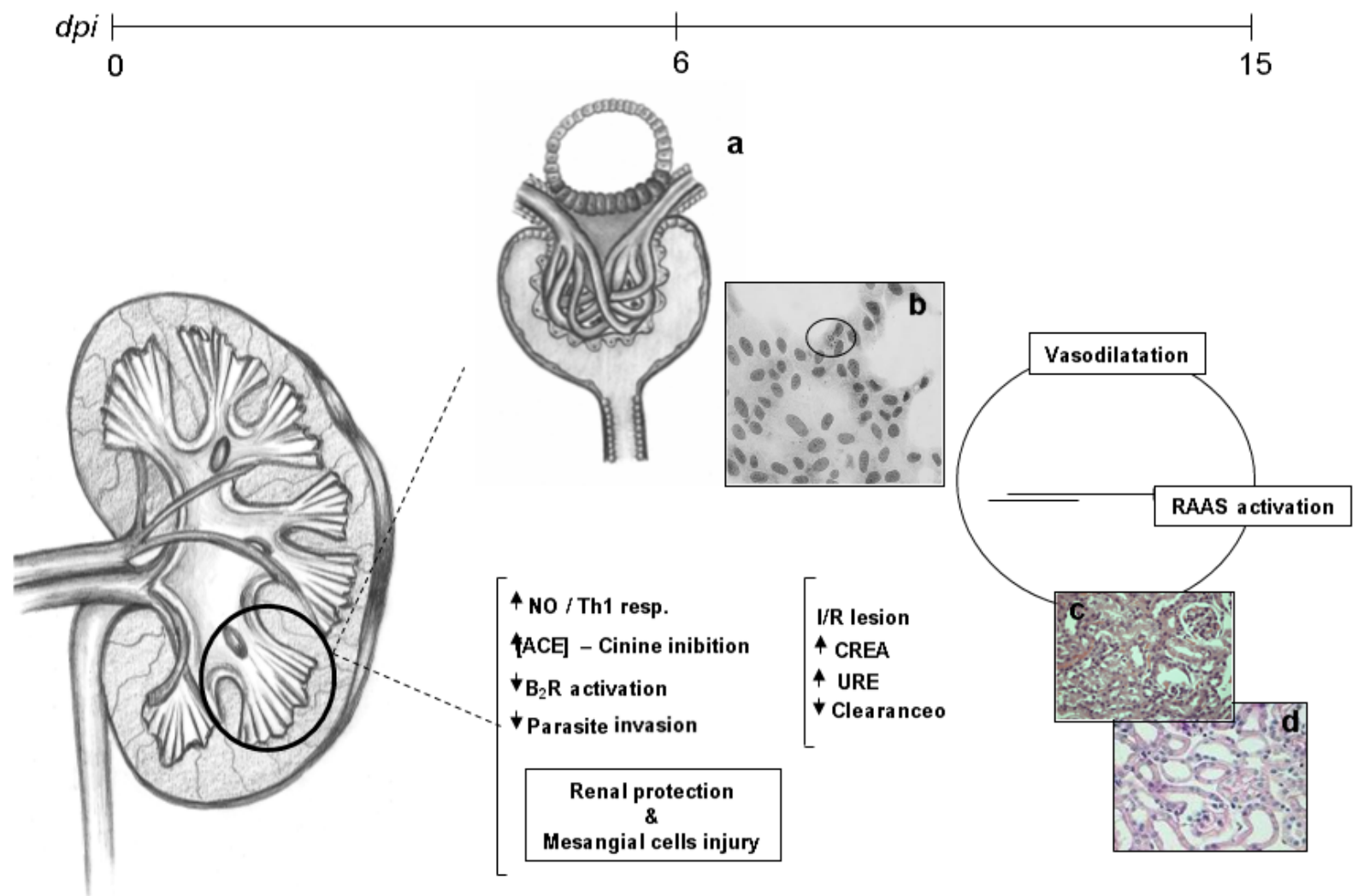

Figure 3. Mechanisms of acute renal injury in mice infected with $T$. cruzi: Between 0 and $15 \mathrm{dpi}$ it is possible to observe the acute kidney injury and the beginning of the connection between the kidney and heart. The presence or some substance secreted by the parasite (chagastoxin) triggers the Th1 pro-inflammatory response through INF-gamma and TNF-alpha cytokines in an attempt to control infection. At this time there is the release of nitric oxide, mainly by the mesangial cells. Nitric oxide (NO), associated with high concentration of angiotensin-converting enzyme (ACE) in the renal endothelium, inhibits the invasion of the parasites in the renal cells (b). This occurs through the microbicidal action of NO, the inhibition of kinins by ACE, and the low activation of type 2 (B2R) bradykinin receptors. At the systemic level, paradoxically, despite the microbicidal action of NO, there is a negative effect of cellular viability and induction of vasodilation that is oberved by the juxtaglomerular apparatus (a) and promoting the activation of the RAAS. We can see in the 6th dpi damage to the proximal tubules and congestion of the capillaries $(c, d)$, in this way there is an elevation of plasma levels of creatinine and urea

disease, the renal involvement has variable gravity and is transitory. We can also set the respective lesion as hidden. The creatinine values may be normal, but they do not indicate that there is no renal impairment, but it will probably still be, is or has already occurred. For these reasons, we consider it essential that the creatinine clearance rate and renal function be monitored during the entire clinical procedure [26].

We believe that the cardio/renal connection is closely linked to the renin angiotensin aldosterone system (RAAS) [27]. However, unlike other models, such as myocardial infarction, this system seems to act in a peculiar way [28].

We blocked the action of RAAS in two different ways through the use of captopril (Captopril 12.5mg, Eurofarma - Brazil), losartan potassium (Losartan 50mg, Germed - Brazil) and spironolactone (Spironolactone 25mg, Eurofarma - Brazil). The use of captopril reduced the mortality of mice susceptible to infection by $30 \%$. Aldosterone blockade reduced this mortality by $40 \%$, but the use of angiotensin receptor blockers (AT1R) increased mortality by $20 \%$ during the acute course of the infection. So, that blockage of RAAS is not the only cause of decreased mortality. The experimental use of captopril is related to anti-inflammatory modulation [29]. In relation to losartan, it seems to us that the positive ionotropic effect of these receptors is necessary for the animal during the course of the acute phase. Aldosterone also appears to be involved in a variety of ways in this model, [30] but one of the beneficial influences we can affirm is the potassium loss, which is very serious ( $15^{\text {th }}$ day after infection) in mice infected with $T$. cruzi (Figure 4).

We used a combination of benznidazole (benznidazole $100 \mathrm{mg}$, Lafepe - Brazil) used for its trypanocidal action and spironolactone in a therapeutic and experimental way. The protocol used was: Benznidazole $(100 \mathrm{mg} / \mathrm{kg})$, orally, once daily associated with spironolactone $(25 \mathrm{mg} /$ $\mathrm{kg}$ ), orally, once daily and for twenty-five consecutive days. We started this treatment on the fifth day after infection, from the visualization of the parasite in the blood and from a slight increase of creatinine in the plasma. Mice with this therapy showed a better physical and clinical condition, such as increased motor activity, exploratory interest, and feed and water consumption when compared to animals receiving benznidazole alone. We found this improvement mainly between the second and third week after infection, which is the period of greatest clinical severity of the disease. Thus, we believe that this association may be effective in improving the quality of life of patients infected by T. cruzi during the acute phase of the disease.

At this time, we are performing the combination of benznidazole, spironolactone and erythropoietin (Alfaepoetina - 4,000 IU, Blausiegel Brazil). Acute renal injury results in decreased erythropoietin production and the presence of the anemic condition [26,31]. We observed marked anemia in infected mice from the tenth day after infection [32]. Thus, 


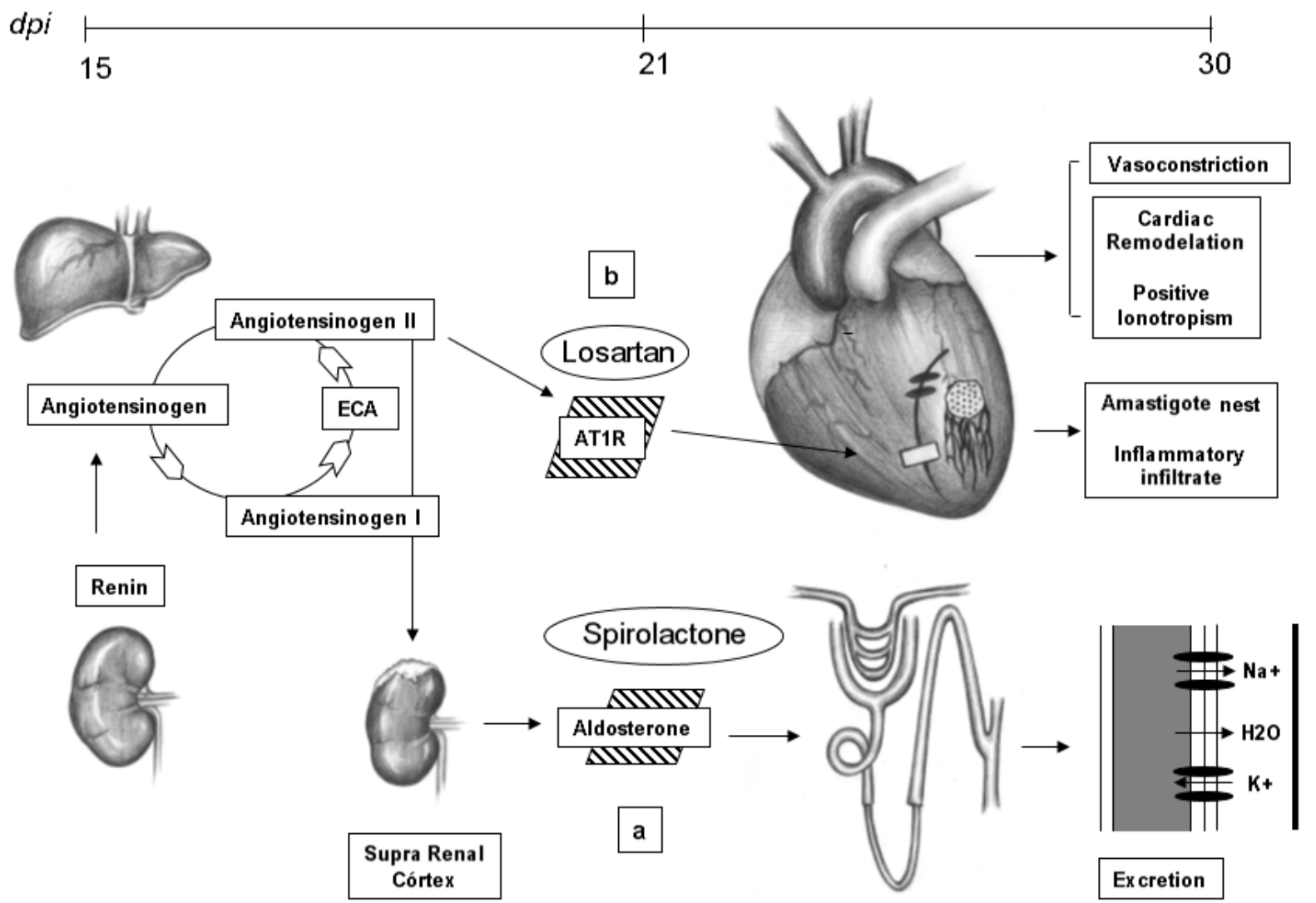

Figure 4. The cardio/renal connection and experimental therapies: Through the activation of the RAAS we can observe two possible pathophysiological mechanisms of the cardio/renal axis. The first is related to the action of aldosterone. Through the use of spironolactone there is a greater retention of potassium, minimizing hypopotassemia (a). Another point is that depending on the modulation, activation of AT1 receptors is required for cardiac remodeling and positive ionotropism of the heart (b). We emphasize that this heart in the $15^{\text {th }}$ dpi is in the moment of greater severity of the acute myocarditis, so if the stimulus of the RAAS in the cardiac muscle is very intense it will aggravate the heart failure and the animal will die

as previously reported, we have the so-called cardio/anemic/renal syndrome, which directly interferes with the quality of life and length of hospitalization of patients with renal insufficiency [33]. In addition, some studies report that erythropoietin has the capacity to minimize acute kidney injury induced by endotoxemia and has beneficial effects in experimental models of septic shock and multiple organ failure $[34,35]$. Therefore, we are carrying out the following experimental therapeutic protocol: from the visualization of the parasite in the bloodstream we administered oral benznidazole in a reduced dosage (100mg/kg - 20 consecutive days) and oral spironolactone (25mg / $\mathrm{kg}$ ) for 25 days consecutive We associate erythropoietin at the dose of $1000 \mathrm{IU} / \mathrm{kg}$ twice weekly, subcutaneously, also from the visualization of the parasites until the end of the acute phase $\left(30^{\text {th }} \mathrm{dpi}\right)$. Our results demonstrate that the decrease in the dosage of beznidazole does not alter the trypanocidal efficacy when compared to the previous protocol $(100 \mathrm{mg} / \mathrm{kg}-20$ consecutive days) and the use of erythropoietin improves the clinical condition of the animals. We are deepening this study in order to evaluate the cardiovascular and renal condition of the infected animals with this reduction in the trypanocidal dosage and the use of the associations.

In summary, Chagas ' disease is a serious public health problem in Brazil and throughout Latin America. It is part of the neglected diseases that do not have great resources or initiatives of pharmaceutical industries in the search of prophylaxis (vaccine) or treatments. From its discovery in 1909 to the present day, many efforts of various research groups try to understand the complexity of this pathology and seek effective ways of treatment and prevention. However, thousands of people are infected annually and thousands more who lose their capacity to perform their duties, have their quality of life compromised and die. Our studies suggest a new aspect of the pathophysiology of T. cruzi infection. We believe that the study of renal injury and the application of associated therapies can be an important focus for improving the treatment of patients infected during the acute phase (Table 1), increasing the quality of life and reducing the side effects of trypanocides used, mainly in children who have a high incidence of adverse reactions to the commonly used treatment.

\section{Ackowledgements}

We would like to thank Drs. Maria de Nazaré Corrêa Soeiro, head of the Laboratório de Biologia Celular (IOC/FIOCRUZ) and Solange Lisboa de Castro for helpful discussions and the logistic support to this research.

\section{References}

1. Chagas C (1909) Nova tripanossomíase humana. Mem Inst Oswaldo Cruz 1: 159-218.

2. WHO (2019) World Health Organization.

3. DNDi (2019) Drugs for neglected disease initiative.

4. MSF on-line (2019) Médicos sem Fronteiras.

5. DataSUS (2019) Ministério da Saúde.

6. Moncayo A (2003) Chagas disease: current epidemiological trends after the interruption of vectorial and transfusional transmission in the Southern Cone countries. Mem Inst Oswaldo Cruz 98: 577-591. [Crossref] 
7. Consenso Brasileiro em Doença de Chagas - Secretária de Vigilância em Saúde do Ministério da Saúde (2006) Rev Soc Bras Med Trop Tropical 3: 124-165. [Crossref]

8. Dias JP, Bastos C, Araújo E, Mascarenhas AV, Martins Netto E, et al. (2008) Acute Chagas disease outbreak associated with oral transmission. Rev Soc Bras Med Trop 41: 296-300. [Crossref]

9. Jansen AM, Xavier SCDC, Roque ALR (2018) Trypanosoma cruzi transmission in the wild and its most important reservoir hosts in Brazil. Parasit Vectors 11: 502. [Crossref]

10. PIDC (2019) Portal de Doença de Chagas - Instituto Oswaldo Cruz.

11. Dias JPC, Coura JR (1997) Epidemiologia. In Dias JPC \& Coura JR (eds) Clínica e terapêutica da doença de Chagas: uma abordagem prática para o clínico terapêutico. 2 th ed. Fiocruz Editora: Rio de Janeiro/Brasil, 33-67.

12. Andréa EM (2004) Doença de Chagas. In Andréa EM (eds) O eletrocardiograma e a clínica. Diagraphic editora: Rio de Janeiro/Brasil, 336-341.

13. Lent H (1999) Evolution of the knowledge about Chagas disease vectors 90 years after its Discovery. Mem. Inst. Oswaldo Cruz 94: 89-92.

14. Teixeira AR, Nascimento RJ, Sturm NR (2006) Evolution and pathology in chagas disease - a review. Mem Inst Oswaldo Cruz 101: 463-91. [Crossref]

15. De Souza AP, Olivieri BP, De Castro SL, Araújo-Jorge TC (2000) Enzymatic markers of heart lesion in mice infected with Trypanosoma cruzi and submitted to benznidazole chemotherapy. Parasitol Res 86: 800-808. [Crossref]

16. Köberle F (1968) Chagas' disease and Chagas' syndrome: the pathology of American trypanosomiaisis. Adv Parasitol 6: 63-116. [Crossref]

17. Lenzi HL, Oliveira DN, Lima MT, Gattass CR (1996) Trypanosoma cruzi: paninfectivity of CL strain during murine acute infection. Exp Parasitol 84:16-27. [Crossref]

18. Melo RC, Brener Z (1978) Tissue tropism of different Trypanosoma cruzi strains. $J$ Parasitol 64: 475-482. [Crossref]

19. Arias LF, Duque E, Ocampo C, Henao J, Zuluaga G, et al. (2006) Detection of amastigotes of Trypanosoma cruzi in a kidney graft with acute dysfunction. Transplant Proc 38: 885-887. [Crossref]

20. Carvalho MF, De Franco MF, Soares VA (1997) Amastigotes forms of Trypanossoma cruzi detected in a renal allograft. Rev Inst Med Trop São Paulo 39: 223-226. [Crossref]

21. Pinto AY, Valente SA, Valente Vda C (2004) Emerging acute Chagas disease in Amazonian Brazil: case reports with serious cardiac involvement. Braz J Infect Dis 8 . 454-460. [Crossref]

22. Kalra V, Mahajan S, Agarwal SK, Tiwari SC (2005) Cardiorenal disease: a clinical intersection. Int Urol Nephrol 37: 175-184. [Crossref]
23. Oliveira GM, Silva T, Batista W, Franco M, Schor N (2009) Acute Trypanosoma cruzi experimental infection induced renal ischemic/reperfusion lesion in mice. Parasitol Res 106:111-120. [Crossref]

24. De Oliveira GM, Yoshida N, Higa EM, Shenkman S, Alves M, et al. (2011) Induction of proinflammatory cytokines and nitric oxide by Trypanosoma cruzi in renal cells. Parasitol Res 109: 483-491. [Crossref]

25. Lemos JR1, Rodrigues WF, Miguel CB, Parreira RC, Miguel RB, et al. (2013) Influence of parasite load on renal function in mice acutely infected with Trypanosoma cruzi. PLoS One 12: e71772. [Crossref]

26. Thadani R, Pascual M, Boneventre JV (1996) Acute renal failure. $N$ Engl J Med $334: 1448-1460$

27. Guyton J \& Hall H. (2004) Fisiologia Cardiovascular In. Guyton J \& Hall H (eds) Human physiology and mechanisms of disease. (10th Edn) Guanabara Koogan SA (Ed) São Paulo/Brasil p. 1156-1602.

28. De Boer RA, PP van Geel, YM Pinto, Suurmeijer AJ, Crijns HJ, et al. (2002) Efficacy of angiotensin II type receptor blockade on reperfusion-induced arrhythmias and mortality early after myocardial infarction is increased in transgenic rats with cardiac angiotensin II type overexpression. J Cardiovasc Pharmacol 39: 610-619. [Crossref]

29. Leon JS, Wang K, Engman DM (2003) Captopril ameliorates myocarditis in acute experimental Chagas disease. Circulation 107: 2264-2269. [Crossref]

30. Salgado DR, Rocco JR, Silva E, Vincent JL (2010) Modulation of the renin-angiotensinaldosterone system in sepsis: a new therapeutic approach? Expert Opin Ther Targets 14: 11-20. [Crossref]

31. Boneventre JV, Zuk A (2004) Isquemic acute renal failure: new inflamatory disease? Kidney Int 66: 480-485.

32. Oliveira G, Masuda M, Rocha N, Schor N, Hooper CS, et al. (2009) Absence of Fas-L aggravates renal injury in acute Trypanosoma cruzi infection. Mem Inst Oswaldo Cruz 104: 1063-1067. [Crossref]

33. Silverberg DS, Wexler D, Blum M, Schwartz D, Wollman Y, et al. (2003) Erithropoitein should be part of congestive heart failure management. Kidney Int Suppl 87: S40-7. [Crossref]

34. Mitra A, Bansal S, Wang W, Falk S, Zolty E, et al. (2007) Erythropoietin ameliorate renal dysfunction during endotoxaemia. Nephrol Dial Transplant 22: 2349-2353. [Crossref]

35. Thiemermann C (2007) Beneficial effects of erythropoietin in preclinical models of shock and organ failure. Crit Care 11:132-135. [Crossref]

Copyright: (C2019 Melo de Oliveira G. This is an open-access article distributed under the terms of the Creative Commons Attribution License, which permits unrestricted use, distribution, and reproduction in any medium, provided the original author and source are credited. 